\title{
Effects of Intercropping with Post-Grafting Generation of Two Ecotypes of Galinsoga parviflora on Quality of Lactuca sativa under Cadmium Stress
}

\author{
Keqiang Li $i^{1, a}$, Liying Ouyang ${ }^{2, b}$, Wanwan Xue ${ }^{1, c}$, Ming'an Liao ${ }^{1, d}$ and Lijin Lin ${ }^{3, e *}$ \\ ${ }^{1}$ College of Horticulture, Sichuan Agricultural University, Chengdu, Sichuan, China \\ ${ }^{2}$ Department of Modern Agriculture, Chendu Agricultural College, Chengdu, Sichuan, China \\ IInstitute of Pomology and Olericulture, Sichuan Agricultural University, Chengdu, Sichuan, China \\ a627563308@qq.com, b24983471@qq.com, c502838055@qq.com, dIman@sicau.edu.cn, \\ ellj800924@qq.com \\ ${ }^{*}$ Corresponding author. Keqiang Li and Liying Ouyang contributed equally to this work.
}

Keywords: Lactuca sativa; Galinsoga parviflora; Intercropping; Post-grafting generation; Quality

\begin{abstract}
The effects of intercropping with two ecotypes of Galinsoga parviflora and their post-grafting generations on quality of Lactuca sativa were investigated through pot experiment under the cadmium stress. The results showed that the intercropping treatment had no significant effect on soluble sugar content in L. sativa, while intercropping with post-grafting generation of $G$. parviflora of mine ecotype as rootstock (MR) decreased the soluble protein content in L. sativa. For the Vc content of L. sativa, intercropping with G. parviflora of farmland ecotype (FCK) had the best effect on its promotion, while intercropping G. parviflora of mine ecotype (MCK) could effectively reduce the nitrate-N content of $L$. sativa leaves. As for the grafting method, the grafting combination of mine ecotypes are used as rootstocks and farmland ecotypes as scions is more effective, intercropping with its post-grafting generation under the cadmium stress had a better effect on the quality of $L$. sativa than on another grafting method.
\end{abstract}

\section{Introduction}

Lactuca sativa, an annual or biennial herb crop, has a high nutritional value as a raw vegetable. However, cadmium (Cd) raises serious concerns as its accumulation in L. sativa not only affect the growth and quality of plant but also threaten the health of consumers [1]. In the application of heavy metal phytoremediation technology in vegetable fields, vegetables can be interacted with hyperaccumulators in heavy metal-contaminated soils. Hyperaccumulators have a stronger ability to absorb cadmium in the soil. On the other hand, in the intercropping process, plants can directly or indirectly have harmful or beneficial effects on other plants by releasing chemical substances to the environment (Allelopathy) [2], and ultimately affect the growth and absorption of heavy metals in soil by vegetables, thus affecting the quality of vegetables. Galinsoga parviflora is a widely distributed Cd-hyperaccumulator [3]. Tang et al. found that Brassica rapa chinensis intercropping with post-grafting generation of Solanum photeinocarpum under cadmium stress, the growth and Cd accumulation of $B$. rapa chinensis increased significantly [4]. This shows that intercropping with post-grafting generation of another plant can affect plant growth and cadmium absorption. Different ecotypes of plants not only show differences in the absorption or tolerance of heavy metals, but also have significant differences in their response to environmental factors [5]. Therefore, in this experiment, the Cd-hyperaccumulator G. parviflora with two ecotypes was grafted, and two ecotypes of $G$. parviflora and their post-grafting generations were intercrossed with $L$. sativa to study their effects on L. sativa quality. In order to screen out the post-grafting generation of G. parviflora that enhances the quality of L. sativa. 


\section{Materials and Methods}

Materials. The seeds of the G. parviflora used in this experiment were collected from farms (farmland ecotype) at Ya'an Campus, Sichuan Agricultural University $\left(30^{\circ} 23^{\prime} \mathrm{N}, 103^{\circ} 48^{\prime} \mathrm{E}\right)$ and Tangjiashan Lead-Zinc Mine (mine ecotype) at Hanyuan County, Ya'an City, Sichuan Province $\left(29^{\circ} 24^{\prime} \mathrm{N}, 102^{\circ} 38^{\prime} \mathrm{N}\right)$. The climatic types and soil physicochemical properties of the two places refer to the results of Lin et al. [6]. The soil samples used in the pot experiment were paddy soil that were collected from the Chengdu campus farm of the Sichuan Agricultural University $\left(29^{\circ} 59^{\prime} \mathrm{N}\right.$, $102^{\circ} 59^{\prime} \mathrm{E}$ ), $\mathrm{pH} 7.35$, organic matter $41.38 \mathrm{~g} / \mathrm{kg}$, total nitrogen $3.05 \mathrm{~g} / \mathrm{kg}$, total phosphorus $0.31 \mathrm{~g} / \mathrm{kg}$, total potassium $15.22 \mathrm{~g} / \mathrm{kg}$, alkaline nitrogen $165.30 \mathrm{mg} / \mathrm{kg}$, available phosphorus $5.87 \mathrm{mg} / \mathrm{kg}$, available potassium $187.03 \mathrm{mg} / \mathrm{kg}$, Cadmium not detected. The basic physical and chemical properties of the soil and the determination of heavy metal content are based on references [7]. $L$. sativa variety is Italian lettuce, with a year-round, resistant to twitching and heat resistance, purchased from Baofeng Seed Company.

Grafting Methods. In October 2015, the two ecotypes of G. parviflora were collected and sowed. When the seedling height is about $3 \mathrm{~cm}$ and two true leaves are spread, they are transplanted into the pot. Afterwards, two ecotypes of $G$. parviflora were grafted when the height of G. parviflora was about $10 \mathrm{~cm}$. The specific grafting method is as follows: (1) Farmland ecotypes are not grafted: The $G$. parviflora seedlings were transplanted directly, and then the seeds were collected for preservation as G. parviflora of farmland ecotype (FCK). (2) Mine ecotypes are not grafted: The G. parviflora seedlings were transplanted directly, and then the seeds were collected for preservation as $G$. parviflora of mine ecotype (MCK). (3) Mine ecotypes are used as rootstocks and farmland ecotypes as scions for grafting: Two ecotypes of $G$. parviflora seedlings were cut from about $6 \mathrm{~cm}$ above the ground. The mine ecotype $G$. parviflora seedlings were used as rootstocks and the farmland ecotype G. parviflora shoots $(4 \mathrm{~cm})$ were grafted as scions. Keep rootstock leaves and buds. After that, collect rootstock seeds as post-grafting generation of G. parviflora of mine ecotype as rootstock (MR) and collect scion seeds as post-grafting generation of G. parviflora of farmland ecotype as scion (FS). (4) Farmland ecotypes are used as rootstocks and mine ecotypes as scions for grafting: Two ecotypes of $G$. parviflora seedlings were cut from about $6 \mathrm{~cm}$ above the ground. The farmland ecotype $G$. parviflora seedlings were used as rootstocks and the mine ecotype $G$. parviflora shoots $(4 \mathrm{~cm})$ were grafted as scions. Keep rootstock leaves and buds. After that, collect rootstock seeds as post-grafting generation of $G$. parviflora of farmland ecotype as rootstock (FR) and collect scion seeds as post-grafting generation of G. parviflora of mine ecotype as scion (MS). During the grafting process, the soil used was free from heavy metal pollution.

Experimental Design. The experiment was conducted in the greenhouse of the Sichuan Agricultural University in Chengdu campus. Air-dried soil $(2.5 \mathrm{~kg})$ was weighed and placed into each polyethylene pot (21 cm high, $20 \mathrm{~cm}$ diamete). Cd was added to the soil samples as analytical reagent $\mathrm{CdCl}_{2} \cdot 2.5 \mathrm{H}_{2} \mathrm{O}$ at the concentration of $10 \mathrm{mg} / \mathrm{kg}$ [4], and then the soil was thoroughly mixed. Natural balance for 4 weeks, occasionally turning soil mixing, so that the soil is fully mixed. In March 2016, the collected G. parviflora seeds and purchased L. sativa seeds were sown and nursed. In April 2016, when the seedlings of $G$. parviflora were about $3 \mathrm{~cm}$ high and 2 true leaves were unfolded, they were transplanted into pots with L. sativa. Seven treatments were applied in the experiment: L. sativa monoculture (CK), intercropping with FCK, intercropping with MCK, intercropping with FR, intercropping with MR, intercropping with FS, intercropping with MS. Monoculture treatment four $L$. sativa per pot, intercropping treatments were planted with three L. sativa and one G. parviflora per pot. Repeat 4 times for each treatment, completely randomized design with $15-\mathrm{cm}$ spacing between pots, timely weeding, pest control, and no disease. After $60 \mathrm{~d}(\mathrm{June} 2016)$, L. sativa leaves are harvested in each pot, and the Soluble sugar [8], Soluble protein [9], Vc [10] and Nitrate-N [11] content were determined. 
Statistical Analyses. Statistical analyses were conducted using SPSS 13.0 statistical software (IBM, Chicago, IL, USA). Data were analyzed by one-way analysis of variance with least significant difference (LSD) at the $\mathrm{P}=0.05$ confidence level.

\section{Results and Discussion}

Soluble Sugar Content in $\mathbf{L}$. sativa. In the Cd-contaminated soil, there was no significant difference in soluble sugar content between intercropping treatments and $\mathrm{CK}(P>0.05$, Fig. 1). Among the seven treatments, L. sativa intercropping with FCK had the lowest soluble sugar content. And $L$. sativa intercropping with MR, had the highest soluble sugar content and was significantly higher than that intercropping with FCK. Compared with intercropping FCK, soluble sugar content in L. sativa intercropping with MR increased by $87.37 \%$.

Soluble Protein Content in L. sativa. Intercropping with two ecotypes of G. parviflora and their post-grafting generations, the soluble protein content in leaves of L. sativa (intercropping with MR) under cadmium stress was significantly lower than that of monoculture, which was reduced by $29.89 \%$ (Fig. 2). However, the soluble protein content in L. sativa leaves in the remaining treatments was not significantly different from the monoculture. For the grafting method, the soluble protein content in L. sativa in intercropping with FR was significantly lower than that of intercropping with FS, and intercropping with MR was significantly lower than intercropping with MS.

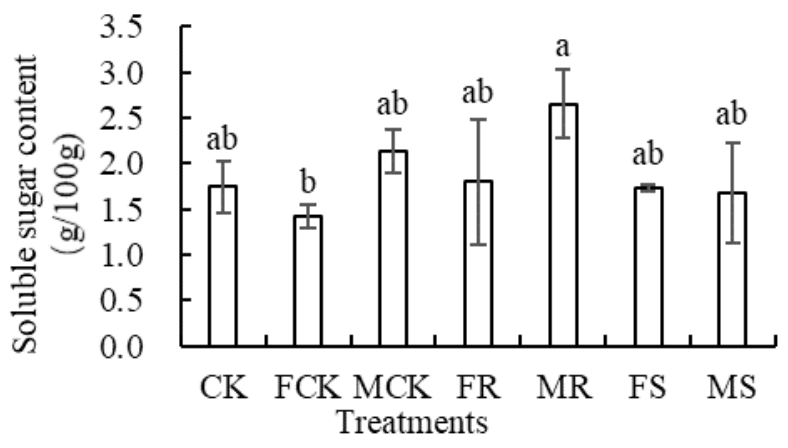

Fig. 1 Soluble sugar content in L. sativa. Different lowercase letters indicate significant differences based on one-way analysis of variance in SPSS 17.0 followed by the least significant difference test $(P<0.05)$.

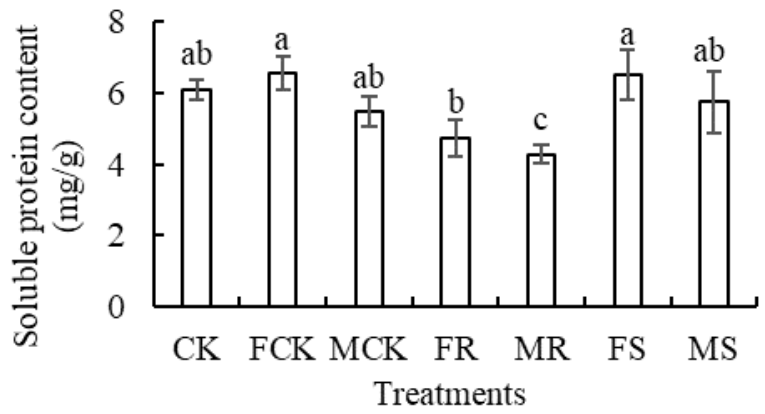

Fig. 2 Soluble protein content in L. sativa. Different lowercase letters indicate significant differences based on one-way analysis of variance in SPSS 17.0 followed by the least significant difference test $(P<0.05)$.

Ve Content in L. sativa. Fig. 3 shows that under cadmium stress, the order of Vc content in L. sativa is: intercropping with FCK > intercropping with FS > intercropping with MS > intercropping with MCK and intercropping with MR $>$ monoculture $>$ intercropping with FR, and the Vc content in $L$. sativa intercropping with FCK, MCK, MR, FS and MS increased by $88.22 \%, 9.11 \%, 12.92 \%$, $62.71 \%$ and $48.38 \%$ compared with the control (monoculture).

Nitrate-N Content in L. sativa. In terms of nitrate-N content, except for intercropping with MS, the nitrate-N content in L. sativa leaves in the intercropping treatment under cadmium stress was significantly lower than that of the monoculture (Fig. 4). Intercropping with MCK had the best effect in all treatments, which resulted in a $67.58 \%$ reduction in nitrate-N content in leaves of L. sativa compared to the monoculture.

\section{Conclusions}

Under the cadmium stress, the quality of $L$. sativa will be adversely affected. After six intercropping treatments, the results showed that intercropping two ecotypes of $G$. parviflora and their post-grafting generations could affect the quality of L. sativa. In general, with the exception of intercropping with 
FR and intercropping with MS, other treatments can improve the quality of $L$. sativa. Among them, intercropping with FCK had the best effect on increasing Vc content, while intercropping with MCK could reduce the nitrate-N content of L. sativa leaves most effectively. As far as the grafting method is concerned, intercropping with MR and intercropping with FS had a better effect on L. sativa quality than intercropping MS and intercropping with FR. This may be due to the better combination of mine ecotypes are used as rootstocks and farmland ecotypes as scions. Therefore, the post-grafting generation of $G$. parviflora had stronger stress resistance, so that more heavy metals could be absorbed during the intercropping process, which reduced the L. sativa stress and finally improved the quality of L. sativa.

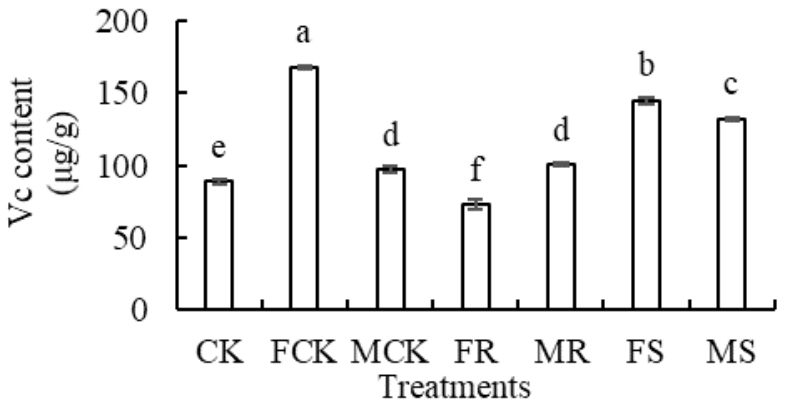

Fig. 3 Vc content in L. sativa. Different lowercase letters indicate significant differences based on one-way analysis of variance in SPSS 17.0 followed by the least significant difference test $(P<0.05)$.

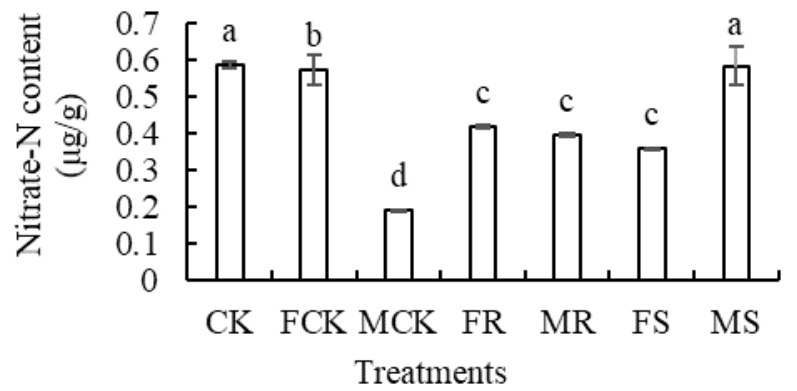

Fig. 4 Nitrate-N content in L. sativa. Different lowercase letters indicate significant differences based on one-way analysis of variance in SPSS 17.0 followed by the least significant difference test $(P<0.05)$.

\section{Acknowledgements}

This work was financially supported by the Application Infrastructure Project of Science and Technology Department of Sichuan Province (2016JY0258).

\section{References}

[1] N. Kaur and S. Jhanji: Journal of Environmental Biology Vol. 37 (2016), p. 993.

[2] M. Farooq, A.A. Bajwa, S.A. Cheema and Z.A. Cheema: International Journal of Agriculture and Biology Vol. 15 (2013), p. 1560.

[3] L. Lin, Q. Jin, Y. Liu, B. Ning, M. Liao and L. Luo: Environmental Toxicology and Chemistry Vol. 33 (2015), p. 2422.

[4] F.Y. Tang, L.J. Lin, D.Y. Yang, M.A. Liao, Q.Q. Ma, C.Y. Liu and J. He: Chinese Journal of Soil Science Vol. 47 (2016), p. 207.

[5] S. Peng, W. Du and Z. Li: Journal of Jishou University Vol. 25 (2004), p. 19.

[6] L.J. Lin, M.A. Liao, L.Y. Mei and J. Cheng: Chinese Journal of Eco-Agriculture Vol. 21 (2013), p. 1565 .

[7] S.D. Bao: Agrochemical Soil Analysis (China Agriculture Press, Beijing 2000).

[8] J.F. Gao: Plant physiology experiment guide (Higher Education Press, Beijing 2006).

[9] Z.L. Zhang: Plant physiology experiment guide (Higher Education Press, Beijing 2016).

[10]L. Li: Plant physiology module experiment guide (Science Press, Beijing 2009).

[11]Q.E. Xiong: Plant physiology experiment course (Sichuan Science and Technology Press, Chengdu 2003). 